Type of the Paper (Article, Review, Communication, etc.)

\title{
Molecular epidemiology characteristics of Brucella abortus strains in China and their relationships with world lineages
}

\author{
Xu-ming Wang ${ }^{1 \ddagger}$, Zhong-zhi Zhao ${ }^{4 \ddagger}$, miao wang ${ }^{3 \ddagger}$, buyun cui ${ }^{2}$, Zhi-guo $\mathrm{Liu}^{2}$ and zhen-jun $\mathrm{Li}^{2}$, \\ 1 Hainan Provincial People's Hospital, Haikou, 570311, China.; Wxmhn1260@163.com \\ 2 State Key Laboratory for Infectious Disease Prevention and Control, National Institute for Communicable \\ Disease Control and Prevention, Chinese Center for Disease Control and Prevention, 155 Chang bai Road, \\ Chang ping, Beijing 102206, PR China; cuibuyun@icdc.cn, wlcblzg@126.com, lizhenjun@icdc.cn \\ 3 Ulanqab Centre for Endemic Disease Prevention and Control, Jining, 012000 Inner Mongolia, PR China.; \\ wangmiao3325@outlook.com \\ 4 Qinghai Institute for Endemic Diseases Prevention and Control, Xining, 811602, PR China; \\ qhzhzhzh@163.com \\ $\ddagger$ These authors contributed equally to this work. \\ * Correspondence: wlcblzg@126.com; lizhenjun@icdc.cn
}

\begin{abstract}
Brucella abortus is a facultative intracellular Gram-negative bacterium that causes chronic persistent infections in humans and livestock. In this study, conventional bio-typing, multiple-locus variable-number tandem repeat analysis (MLVA), and whole-genome sequencing-single-nucleotide polymorphism (WGS-SNP) were used to investigate the molecular epidemiology characteristics of Brucella abortus strains in China and their relationships to world lineages. A total of 100 strains were collected from 1953 to 2013, suggesting that B. abortus circulated in China in the past five decades. Moreover, most strains were mainly distributed in the northwest area. During this period, seven biovars were found, indicating that $B$. abortus displayed a high diversity of biovars and that the main epidemic areas are in the northern provinces. Strains have high genetic diversity, and bruce07 is the most helpful locus for genotyping of this population. Moreover, 17 MLVA-11 genotypes were found; 13 of them are of known genotypes and four are unassigned genotypes, indicating that $B$. abortus in this study had several geographic origins. Still, strains from unassigned genotypes may originate from China. Many shared MLVA-16 genotypes were observed in strains from the same provinces in Northern China, which confirmed a brucellosis outbreak within northern regions. WGS-SNP analysis showed that eight Chinese strains formed a ladder-like phylogram (C. VII) with strains from nine countries, including Uganda, Iraq, Russia, Georgia, Spain, Italy, Egypt, Mongolia, and China; strains were introduced to these countries from a single source. Notably, Chinese strains were closely related to strains from Russia and Mongolia; frequent animal (cattle) trade and exchanges may promote this process. We will provide new and insightful information for strength surveillance and control of B. abortus brucellosis in China.
\end{abstract}

Keywords: B. abortus; molecular epidemiology; MLVA; WGS-SNP; China

\section{Introduction}

Brucellosis, one of the world's most common and significant zoonoses, affects various domestic and wildlife animals, and also humans [1]. Abortion and infertility in the infected animals cause hung economic loss, whereas prolonged low fever, fatigue, and sweat in humans resulted in significant public health concerns worldwide [2]. Bovines and small ruminants, which excrete bacteria in the milk and reproductive discharges, are major sources of infection for humans and other animals [3]. Contact with infected domestic and consumption of raw dairy products are the main routes for human infection [4]. Brucellosis is caused by species of the genus Brucella, which includes B. abortus, B. melitensis, B. suis, B. ovis, B. canis, and B. neotomae [5]. Of these, B. abortus, B. melitensis, and B. suis 
are the most vital agents for human brucellosis in China [6]. Our previous study showed that B. melitensis is a latent "travel bacterium" that spread and expanded from North China to South China [7]. However, the impact on the public health and development of B. abortus' animal husbandry should never be overlooked [8]. In Northern China, where the traditional agropastoral areas with more developed animal breeding industries are located, the brucellosis seroprevalence was greater than 10\%; , the seroprevalence of brucellosis in Southern China reached only 5.5\% [9]. Despite the relatively low seroprevalence $(2.8 \%)$ in a serological survey of yaks, the estimated economic losses were substantial in Tibet. With no control program for brucellosis in yaks, the total annual economic loss in the study area was estimated at US\$521, 043, mainly resulting from abortions and reduction of the milk and meat yield [10]. However, there is limited available information on the molecular epidemiology of brucellosis caused by B. abortus.

A bio-typing assay is time consuming and difficult to interpret due to limited standardization of the typing reagents, but it was useful for Brucella strain identification and species differentiation [11]. Moreover, multi-locus variable-number tandem repeat assays (MLVA) are a genetic approach with high discriminatory power in the Brucella genus, providing a fine-scale resolution among isolates and allowing the determination of the source of infection, geographical origin, and spread pattern of disease [12,13]. The whole-genome sequencing-single-nucleotide polymorphism (WGS-SNP) approach is used to establish phylogenetic relationships of Brucella isolates [14,15]. In this study, 100 strains were isolated from samples collected between 1953 and 2013 from various host animals throughout the countrywide in China. This study characterized these Brucella strains using biotyping, MLVA, and WGS-SNP, to determine the distribution regions, genotyping characteristics, geographic origins, and phylogenetic relationships of B. abortus strains at the global level. The findings could support a nation-wide control and prevention program against brucellosis.

\section{Materials and Methods}

\subsection{The source of Bacterial strains}

The MLVA-16 characteristics, panel1, MLVA-11 genotypes, species/biovars, hosts, location, and year of B. abortus strains were collected, and Microsoft Excel (Microsoft, Redmond, CA, U.S.) was used for data cleaning. A total of 100 strains were obtained in this study; 65 were found in cattle, 20 in humans, 7 in sheep, 4 in yak, 3 in goat, and for 1 strain the host is unknown. Moreover, 36 strains of previously published from Inner Mongolia [16] were involved in this study. The isolates and biovar of the strains were identified using standard procedures [17]. All isolates were identified as Brucella species based on morphology and conventional identification methods according to standard biotyping procedures, including the need for $\mathrm{CO} 2$ for growth, $\mathrm{H} 2 \mathrm{~S}$ production, sensitivity to thionin (10 and $20 \mu \mathrm{g} / \mathrm{ml})$, basic fuchsin $(20 \mu \mathrm{g} / \mathrm{ml})$, and agglutination with mono-specific antiserum for and $\mathrm{M}$ antigens and phage lysis test (Tbilisi, Tb; Berkeley, Bk2; Weybridge; Wb) $[18,19]$. AMOS-PCR (B. abortus bv. 1, 2, and 4, B. melitensis, B. ovis, and B. suis bv. 1) [20] was used to verify further B. abortus bv. 1, 2, and 4 .

\subsection{Brucella MLVA-16 genotyping scheme}

MLVA-16 in all strains was performed as previously described [21,22]. Briefly, the 16 primer pairs were divided into three panels: panel 1 (MLVA-8: eight loci including bruce06, bruce08, bruce11, bruce12, bruce42, bruce43, bruce45, and bruce55), often used for species identification, panel 2A (three loci including bruce18, bruce19, and bruce21), and panel 2B (five loci including bruce04, bruce07, bruce09, bruce16, and bruce30), used for genotype comparison; MLVA-11 (panels 1 and 2A) (trace-back geographic origin of strains), and MLVA-16 (panels 1, 2A, and 2B). Fragments were sized after comparison with a ROX (carboxy-X-rhodamine)-labeled molecular ladder (MapMaker 1000; Bioventures Inc., Murfreesboro, TN, USA) and Gene Mapper software version 4.0 (Applied Biosystems). The fragment sizes were subsequently converted to repeat unit numbers using a published allele-numbering system [23].

\subsection{Analysis of the MLVA data}

The genetic diversity of $B$. abortus strains in this study was calculated based on the Hunter-Gaston diversity index (HGDI) according to a previously published method [24]. BioNumerics version 5.1 
software (Applied math, Belgium) was used to analyze the MLVA-16 assay data (Table S1). Both the categorical coefficient and unweighted pair group methods were applied to the clustering analysis. MLVA-11 was used to investigate the geographical origins between our isolates [25,26] and 1,482 isolates from the MLVA bank (http://microbesgenotyping.i2bc.paris-saclay.fr/databases) (Table S2), and minimum-spanning trees were constructed using the goeBURST algorithm with PHYOVIZ 2.0 [27]. The MLVA-16 approach was applied to the genetically related investigation between strains from those reported in China ( $\mathrm{n}=100)$ (Table S1) and at the global level $(\mathrm{n}=1525)$ (Table S3). Moreover, 62 B. abortus (Table S4) genomes were retrieved from GenBank and used for genomic comparison and phylogenetic analyses. The phylogenetic tree was constructed using the TreeBeST [28] based on maximum-likelihood phylogenies (PHYML) with 1,000 bootstrap replicates.

\section{Results}

3.1. The species/biovars, region distribution, and host profile of Chinese B. abortus

A total of 100 B. abortus samples were collected from 1953 to 2013 (11 in the 1950s, 9 in the 1960s, 5 in the 1970s, 38 in the 1980s, 1 in 1990, 4 in 2000s, and 32 in 2010s). A total of seven biovars were observed in this population, including B. abortus bv. $1(\mathrm{n}=24)$, B. abortus bv. $2(\mathrm{n}=2)$, B. abortus bv. 3 $(\mathrm{n}=60)$, B. abortus bv. $5(\mathrm{n}=1)$, B. abortus bv. $6(\mathrm{n}=2)$, B. abortus bv. $7(\mathrm{n}=1)$, and B. abortus bv. $9(\mathrm{n}=$ 10). B. abortus bv. 3 was the dominant species in China (Table 1). These strains were collected from 14 provinces (cities and autonomous regions) from 1953 to 2013, including 36 strains in Inner Mongolia, 14 in Xinjiang, 11 in Hebei, 10 in Heilongjiang, 6 in Gansu, and 5 in Zhejiang; the remaining regions contained 1 to 4 strains, $85 \%$ strains was distributed in northern, China. Moreover, 100 strains were obtained from five hosts, including 65 in cattle, 65\%, followed by 20 in humans, 7 in sheep, 4 in yak, 3 in goat, and 1 in an unknown host (Table 1).

3.2. MLVA-16 genotyping results

Based on HGDI, three loci (bruce06, 08, and 12) showed no diversity (HGDI=0.0000) and bruce55 showed the highest diversity in all loci of panel 1, with a value of HGDI of 0.6515 . The lowest value of HGDI in panel 2B was bruce16 (HGDI $=0.2576$ ). The HGDI value in other four loci were $>0.5705$, and the HGDI value of panel1, MLVA-11, and MLVA-16 was 0.7820, 0.8196, and 0.9853, respectively (Table 2). In panel 1, 10 genotypes were observed, of which 7 are known, including $116(n=1), 30$ (n $=4), 37(\mathrm{n}=5), 112(\mathrm{n}=13), 117(\mathrm{n}=15), 28(\mathrm{n}=17)$, and $36(\mathrm{n}=39)$. The remaining three unassigned genotypes were Ba8-1 $(n=2)(4-5-3-12-2-1-3-2)$, Ba8-2 $(n=2)(4-5-3-1-2-3-1-3-2)$, and Ba8-3 $(n=2)(4-$ 5-3-1-2-3-1-5-2) (Fig. 1).

Bases on MLVA-11, 17 genotypes were found, 13 of which were of known genotypes, namely, $71(\mathrm{n}=1), 83(\mathrm{n}=1), 181(\mathrm{n}=1), 210(\mathrm{n}=9), 331(\mathrm{n}=1), 327(\mathrm{n}=3), 75(\mathrm{n}=4), 78(\mathrm{n}=4), 326(\mathrm{n}=4), 77$ $(n=5), 82(n=11), 328(n=12), 72(n=38)$, and 4 novel genotypes: Ba11-1 $(n=1)$, Ba11-2 $(n=1)$, Ba11$3(n=2)$, and Ba11-4 (n=2) (Fig. 1). Genotype 72 was distributed in seven regions, the higher diversity of MLVA-11 genotype was observed in strain from Inner Mongolia, Xinjiang Province, Hebei Province, and Sichuan Province (Table 1). However, the novel MLVA-11 genotype was found in Ningxia, Heilongjiang Province, and Hebei Province (Table 1). Based on the complete MLVA-16 loci, 100 strains were divided into four groups (I-IV) and 65 MLVA-16 genotypes (G.T.) had a 50\% similarity coefficient. Both biovar 1 and 2 fell into group I; the other five biovars 3, 5, 6, 7, and 9 were clustered in other three groups (I, III, and IV), of which group I contained six subgroups (a-f) (Fig. 1). The 54 strains consisted of 19 shared genotypes that contained 2-8 strains, and 46 strains of an independent genotype, each present a single strain. Eighteen out of 19 strain-shared genotypes comprised strains from the same province; only one shared genotype (GT48) contained five strains obtained from four provinces (Fig. 1).

3.3. Geographic origin profile of Chinese B. abortus strains on a global scale

Among 17 MLVA-11 genotypes, seven were shared genotypes (72, 75, 82, 83, 181, 210, and 328) comprising strains from 2 to 10 countries, and the remaining 10 genotypes were single genotypes that were exclusively found in Chinese strains. The genotype $72(\mathrm{n}=38)$ was the predominant population and accounted for 38\% (38/100), followed by genotype $328(\mathrm{n}=12), 82(\mathrm{n}=11)$, and $210(\mathrm{n}=9)$, accounting for $12 \%(12 / 100) 11 \%(11 / 100)$, and $9 \%(9 / 100)$, respectively. MLVA-11 genotype 72 was 
shared by strains from six countries, including France, Germany, Italy, Kazakhstan, Portugal, and China. Genotype 82 comprised strains from 10 countries, including Brazil, Costa Rica, France, Germany, Italy, Kazakhstan, Portugal, South Korea, the United States, and China. Genotype 210 consisted of strains from four countries, Costa Rica, Italy, United States, and China. Genotype 78 was shared by strains from England, the United States, and China. Genotype 83 was shared by strains from Brazil, Costa Rica, England, and China. Genotype 75 included strains from Brazil and China. Genotype 181 consisted of strains from Portugal and China. However, another 10 single genotypes were exclusive of strains from China (Fig. 2).

3.4. Worldwide phylogenetic analysis based on both MLVA-16 and WGS-SNP

The MLVA-16 comparison analysis showed that 1,625 strains were sorted into three groups (AC), and Chinese strains (group A) were clustered together with strains from Italy, Kazakhstan, and Brazil, but had no sharing of the MLVA-16 genotype was observed (Fig. 3). The whole-genome SNP analysis showed that 65 worldwide B. abortus strains were grouped into seven clades ( I -VII) (Fig. 4); 16 Chinese strains were divided into five clades (Clade I, III, IV, VI, and VII). Clade I was composed of two Chinese strains and one strain from Kenya; clade III included strains from China, Poland, and Spain; clade IV included strains from China, South Korea, and USA; clade VI included strains from China, Italy, and Greece. Clade VII was composed of 19 strains from nine countries, including Uganda, Iraq, Russia, Georgia, Spain, Italy, Egypt, Mongolia, and China. These strains formed a ladder-like phylogram. However, most strains $(n=8)$ from China were found in clade VII and were the most similar to strains from Russia and Mongolia (Fig. 4). 


\subsection{Figures, Tables and Schemes}

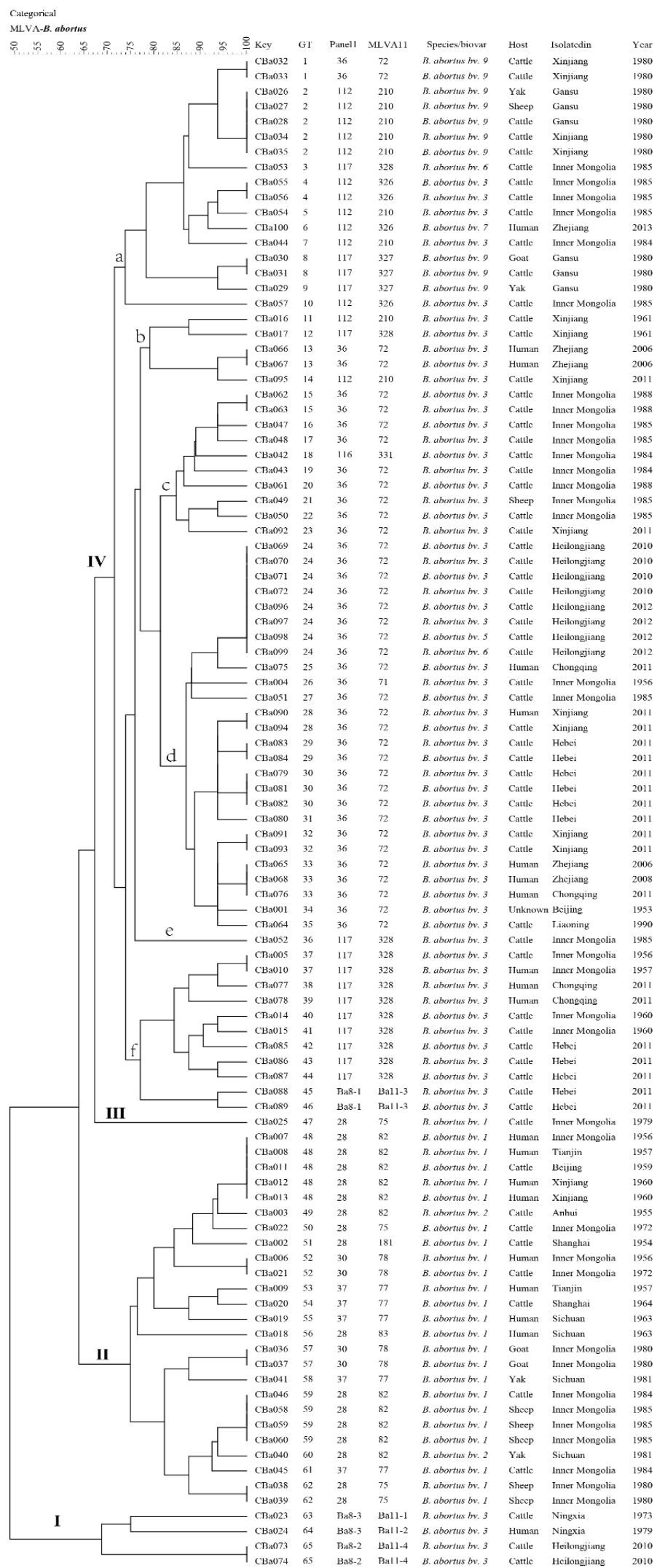

Figure 1. Dendrogram based on the MLVA-16 genotyping assay (UPGMA method) showing the relationships among the $100 \mathrm{~B}$. abortus isolates. The columns show the identification numbers (Key), MLVA-16 genotypes (GT), MLVA-11 (panels 1 and 2A) genotypes, species biovars, hosts, geographical locations, and the year each strain was isolated. 


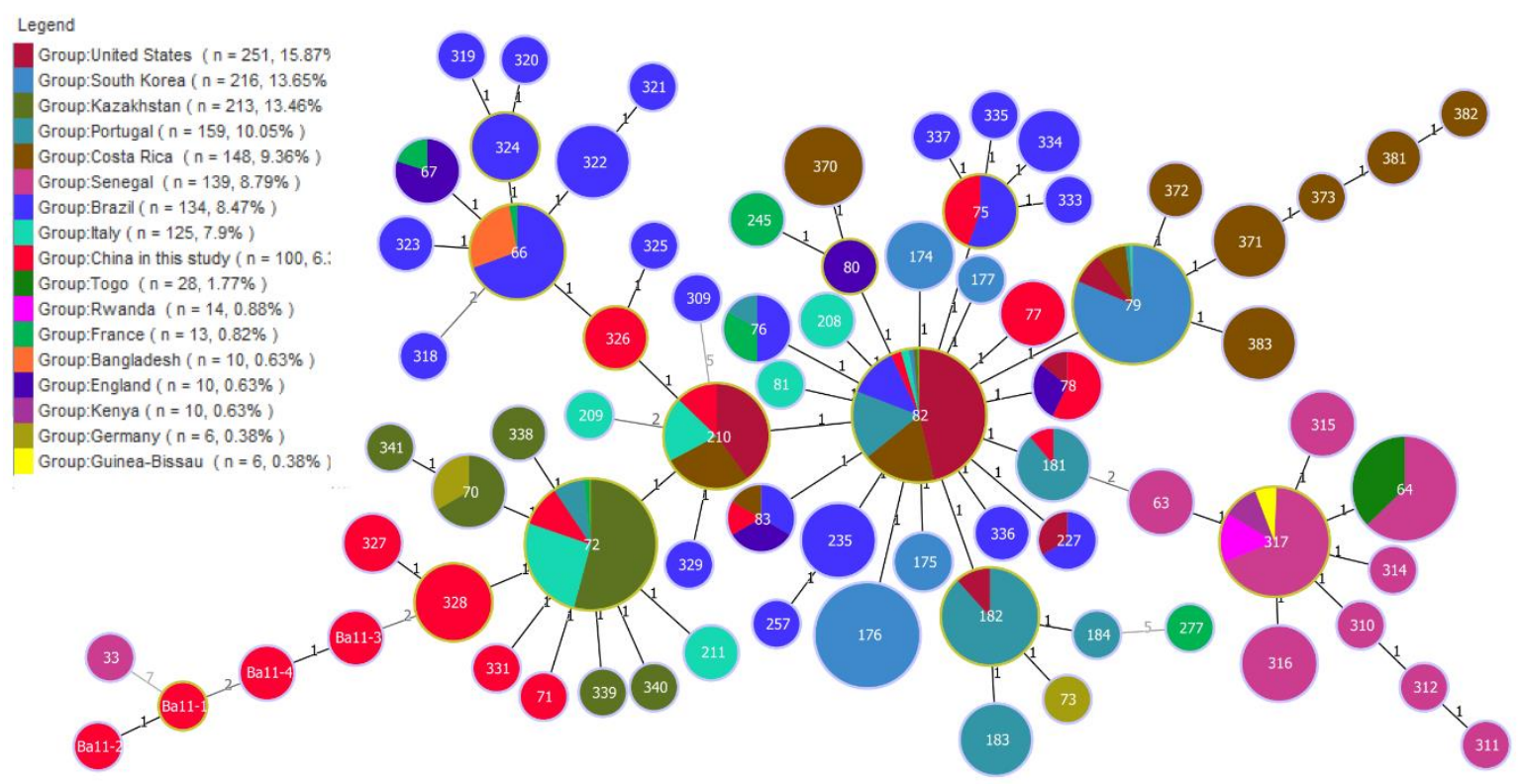

Figure 2. Geographic origins of Chinese B. abortus strains based on MLVA-11.

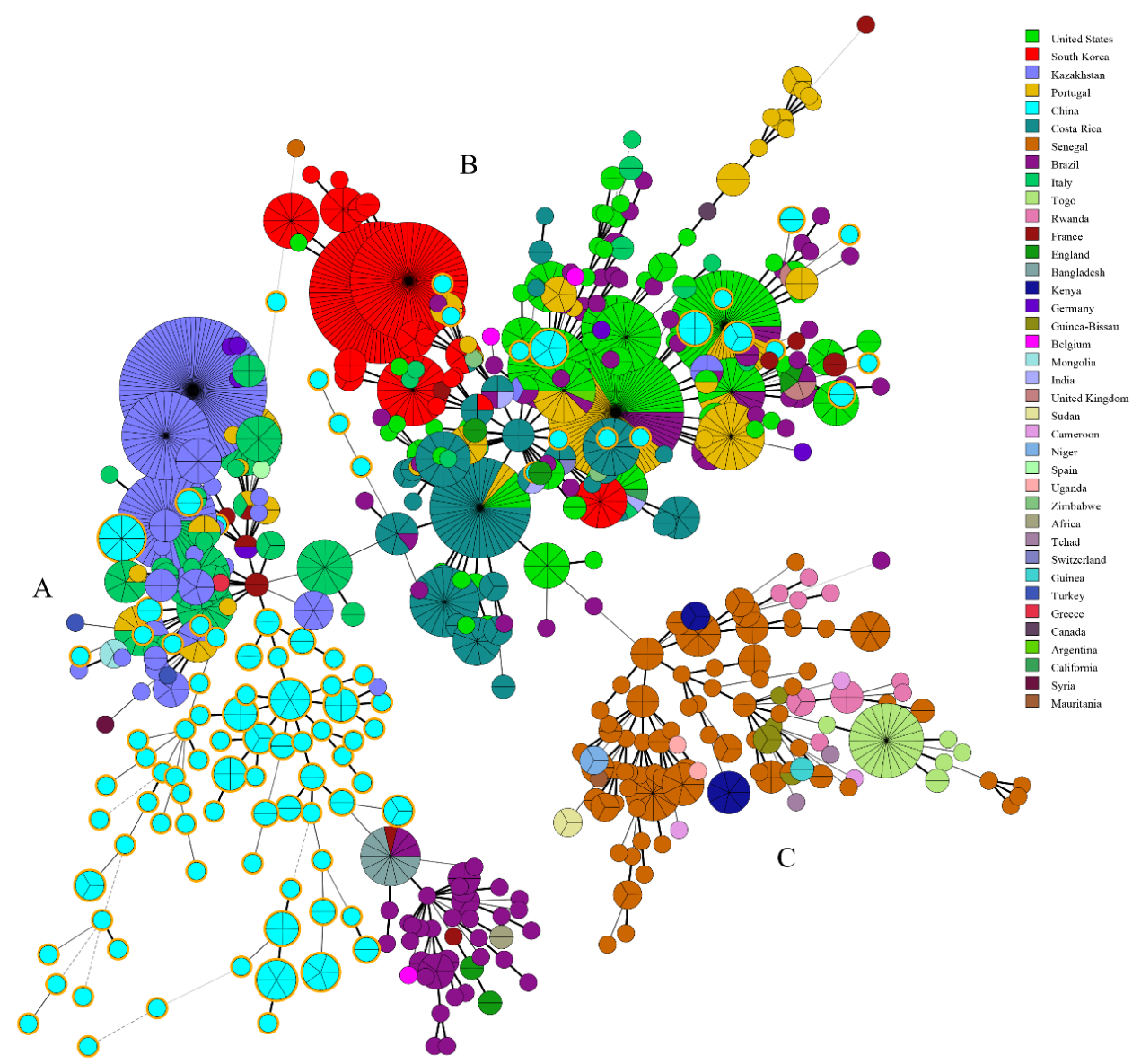

Figure 3. Minimum spanning tree for B. abortus using MLVA-16 data with Chinese isolates (blue). 


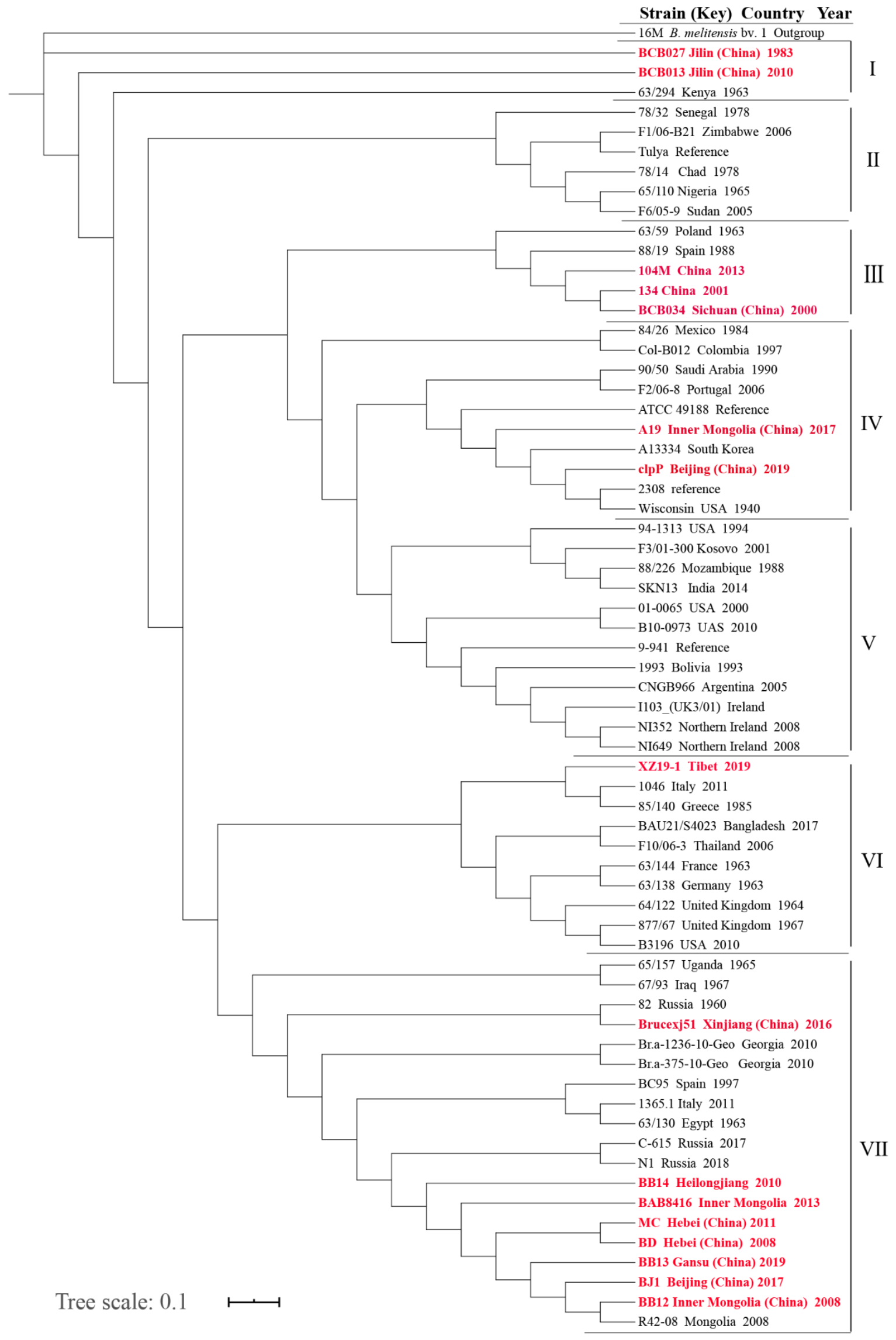

Figure 4. WGS-SNP phylogenetic analysis of B. abortus at a global level. 
Table 1. Location, numbers, percentages (\%), species, panel1, MLVA-11, hosts, and year of Brucella abortus in this study.

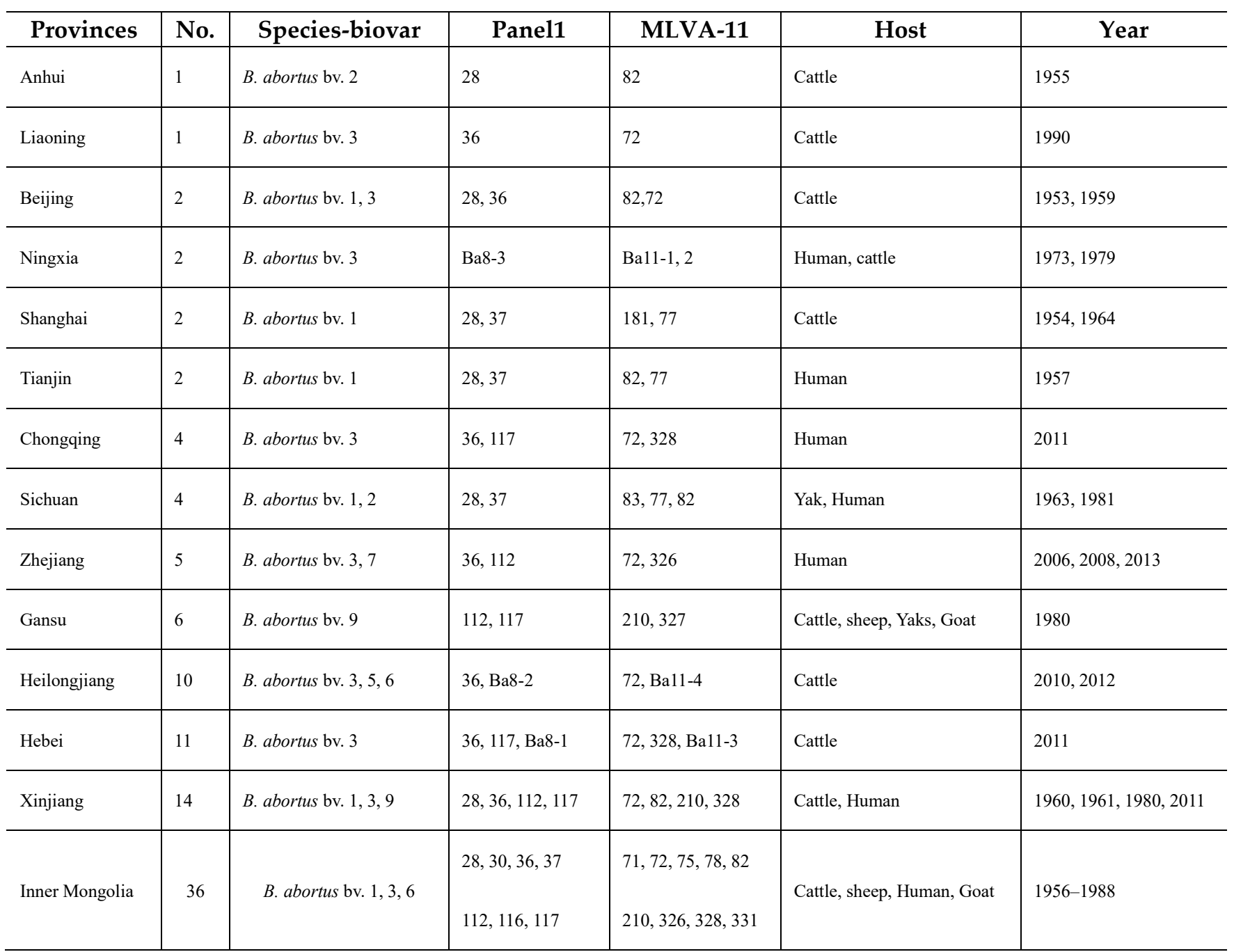

Table 2. Allelic types and HGDI of B. abortus for 16 loci in this study.

\begin{tabular}{cc}
\hline Panel and Locus & HGDI values \\
\hline Bruce06 & 0.0000 \\
Bruce08 & 0.0000 \\
Bruce11 & 0.4034 \\
Bruce12 & 0.0000 \\
Bruce42 & 0.0776 \\
Bruce43 & 0.1139 \\
Bruce45 & 0.0396 \\
Bruce55 & 0.6515 \\
Bruce18 & 0.1701 \\
Bruce19 & 0.2204 \\
Bruce21 & 0.0396 \\
Bruce04 & 0.6392 \\
Bruce07 & 0.7905 \\
Bruce09 & 0.7719 \\
Bruce16 & 0.2576 \\
Bruce30 & 0.5705 \\
Panel 1 & 0.7820 \\
MLVA-11 & 0.8196 \\
MLVA-16 & 0.9835 \\
\hline
\end{tabular}




\section{Discussion}

B. abortus infection was highly endemic in dairy herds in China [9]. The comprehensive molecular epidemiological and phylogenetic analysis of the $B$. abortus strains nationwide are useful for improving the surveillance and control program to determine the risk factors associated with brucellosis in humans. In this study, 100 B. abortus were collected from 1953 to 2013, indicating that $B$. abortus strains have circulated in China for the past several decades, but the strain numbers were lower than those of B. melitensis [29]. This observation is in agreement with a report that B. abortus was a secondary pathogen agent for brucellosis in China [6]. The seven biovars were observed in this study, and B. abortus bv. 3 was dominant species in China, suggesting that B. abortus in China has had a high diversity of species/biovars, which poses a potential challenge for animal vaccination. The $85 \%$ strains were distributed in traditional and domestic animals breeding developed provinces, providing helpful information for disease control and prevention. However, the distribution range of this species is different from that of the B. melitensis strains, which cover all mainland regions in China [30]. Similarly, the most frequently recorded B. abortus biovar is biovar 3 in the Republic of Kazakhstan. More than $90 \%$ of the overall B. abortus samples were isolated from the northern regions of East and West Kazakhstan [31]. Five kinds of hosts were observed in this population, and $65 \%$ of strains were from cattle. This indicated a narrower host spectrum than that of B. melitensis [32], which is a significant impact factor for strains with similar geographic distribution and is closely related to the low virulence of $B$. abortus strains. In Italy, the strains identified from cattle showed a high prevalence of B. abortus bv. 3 isolates (84.5\%), followed by B. melitensis bv. $3(9.9 \%)$ and B. abortus biovars 1 and 6 (5.5\% and $0.1 \%$, respectively) [33]. In Africa, B. abortus bv. 3 is the most commonly isolated strains in cattle [[34]. Moreover, there were no strains from swine and wild animals; further pathogen surveillance in other hosts is a priority.

The complete MLVA-16 approach exhibited excellent discriminatory power to B. abortus with a high HGDI value (0.953). Notably, five panel 2B markers had a high Hunter-Gaston index, and bruce 07 is the most helpful locus for genotyping B. abortus in China. This result was consistent with B. canis data in China; there were at least two alleles for each of the 16 loci investigated by the MIVA approach [12]. However, this feature is different from the results of studies of B. melitensis diversity in China. The majority of loci from panel 1 and panel 2A showed low diversity, and the bruce04 locus displayed the highest diversity (HGDIs $=0.841$ ) [35]. MLVA-16 panel has a high discriminatory power to B. abortus from Italy, with polymorphism levels of 0.939 based on the HGDI, compared to 0.380 and 0.380 for the MLVA- 8 and MLVA-11 panels, respectively; and the highest variability was detected in the 2B panel for the bruce09 locus [36]. HGDI analysis of B. abortus bv. 3 from West Africa showed that the highest diversity indices were observed with markers composing panel 2 with three to nine alleles, especially at bruce16, which is known as one of the most variable loci [37]. Betsy J et al. reported that, historically, approximately $85 \%$ of B. abortus infections in the U.S. were caused by biovar 1, which would result in an HGDI $\approx 0.2$ [38]. The variability of HGDI features among strains from different regions may reveal different microevolution patterns of the Brucella population [39], suggesting that the strains described in this study were introduced from multiple geographic regions.

A total of 17 MLVA-11 genotypes were observed; the higher diversity of MLVA-11 genotype was observed in strains from Inner Mongolia, Xinjiang Province, Hebei Province, and Sichuan Province, suggesting that animal trade often occurred among these regions in the past. The seven shared MLVA-11 genotypes $(72,75,82,83,181,210$, and 328) comprising strains from 2 to 10 countries suggest that the strains from each shared genotype had a common source, indicating that the $B$. abortus from this study had multiple geographic, cross-border transmission, probably due to exchange and trade of infected animal. Trade ties and livestock exchange between countries have a long history; archeological findings unraveled trade relations between the nomadic people who inhabited Central Asia and China long before the Common Era (C.E.) [40]. MLVA-11 genotype 72 was shared by strains from six countries, including France, Germany, Italy, Kazakhstan, Portugal, and China. MLVA-16 comparison analysis showed that Chinese strains were clustered together with Italy, Kazakhstan, and Brazil. A previously reported that, 4,500 years ago, seasonal nomadic pastoralist routes were formed from modern Southern Kazakhstan to Xinjiang Uygur Autonomous 
Region, covering more than $70 \%$ of the high-mountain route of the silk road [41]. However, there is no trade-in live cattle between Kazakhstan and China in modern history, but actively developing livestock products [42]. Furthermore, four strains from Sichuan Province had three MLVA-11 genotypes (83, 77, and 82), two of which were shared (82 and 83 ) with strains from other regions, and one (77) is exclusive of strains from Sichuan Province. Although it was a historical area of B. abortus brucellosis, it had no shared genotype 72 [6]. The investigation of serology and bacteriology of $B$. abortus infection in this region is a priority. However, 10 single genotypes were exclusively found in Chinese strains, indicating that these strains originated from China lineages, but further investigation on the origin of the strains is recommended.

In this study, the 19 shared MLVA-16 genotypes included 54 strains, with each genotype representing 2-8 strains, and 18 out of 19 shared genotypes consisted of strains of the same province. Only one shared genotype (GT48) was present in five strains, and these were obtained from four different provinces, suggesting that the majority of brucellosis cases were outbreak epidemic within respective regions. This conclusion is in agreement with the geographic distribution of the strains and outbreak cases mainly focus on Northern China. Moreover, the remaining 46 independent genotypes of each single strain indicated that $46 \%$ (46/100) cases had epidemiology unrelated or sporadic characteristics [43]. Strengthening the surveillance of B. abortus animal infection applied serology, bacteriology, molecular assays in northern regions, China helps better understand the epidemiology of brucellosis.

The sixteen Chinese strains were divided into five clades by whole-genome SNP analysis, confirming that strains from this study had multiple ancestor strains. Remarkably, clade VII formed a ladder-like phylogram and consisted of strains from nine countries, including Uganda, Iraq, Russia, Georgia, Spain, Italy, Egypt, Mongolia, and China from a single ancestor. The B. abortus isolates from Italy are substantially different from those found in Europe and North America and are more closely related to strains from the Middle East and Asia [44]. Furthermore, most Chinese strains $(n=8)$ were the most similar to Russia and Mongolia; they were geographically close. They revealed that strains from a common ancestor were continuously circulating among these countries. Human brucellosis seroprevalence among rural people in Mongolia is high [45], and Mongolian B. melitensis isolates had high genetic similarity with Chinese strains, likely due to geographical proximity [46]. Moreover, some strains from Mongolia had closely related MLVA genotypes to strains from Russia [47]. In the North Caucasian Federal District, the largest number of new human brucellosis cases was notified in the Republic of Dagestan (59.3\%) and the Stavropol Territory $(27.4 \%)$, and the true prevalence of brucellosis greatly exceeds the official statistical data [48]. The frequent spread of these lineages from one country to another due to long-term trading partnerships between the three countries is a likely explanation for the data. Otherwise, due to the uncontrolled introduction of the agents via humans, infected animals, semen, and vectors have a high risk of B. abortus infection [49]. In endemic countries, combining the serodiagnosis and bacteriology, and molecular diagnosis for surveillance of brucellosis is essential. Molecular genotyping should be systematically applied to support control plans for control of brucellosis in China.

Moreover, our study has some limitations. First, the strains were collected from previous studies that might have been influenced by many aspects, such as the local economic situation and the diagnosis and surveillance status of brucellosis. Second, due to variability in the number of strains collected among different provinces and for different years in this study, a genome analysis of strains from more regions and hosts is recommended. Third, no related epidemiology data were collected, and analysis of animals moves, and exchange is lacking. Therefore, a whole-province survey on human and animal infections with B. abortus should be initiated. 


\section{Conclusions}

In this study, a nationwide molecular analysis of B. abortus strains were performed. Our research showed that there was a high diversity of biovar and MLVA-11 genotypes among this population. $B$. abortus strains exhibited multiple geographic origin characteristics and the predominant genotype was shared with strains from many countries, indicating that the strains have multiple geographic origins. Chinese strains were similar to strains from Russia and Mongolia. These have been traditionally countries of cattle and sheep breeding industries. Our work contributes to a better understanding of the epidemiology of B. abortus brucellosis in China and also provides invaluable information that could be helpful to devise control strategies for the disease.

Supplementary Materials: The following are available online at www.mdpi.com/xxx/s1, Figure S1: title, Table S1: title, Video S1: title.

Author Contributions: LZG and ZZZ performed strains collected and analysis, WXM and LZG performed genotyping and cluster analysis and drafted the manuscript; WM conducted epidemiological investigations and data analysis; CBY and LZG participated in the design of the study and critically reviewed the manuscript; WXM and LZJ participated in the design of the study and managed the project. All authors read and approved the final version of the manuscript.

Funding: This study was supported by the National Key R\&D Program of China, grant number 2018ZX10734401, 2018ZX10734404, and 2017ZX10303401. The funding agencies had no role in the study design, data collection and analysis, decision to publish, or preparation of the manuscript.

Conflicts of Interest: The authors declare no conflict of interest. The funders had no role in the design of the study; in the collection, analyses, or interpretation of data; in the writing of the manuscript, or in the decision to publish the result.

\section{Appendix A}

Abbreviations

PCR: Polymerase chain reaction

MLVA: Multiple-locus variable-number tandem repeat analysis

VNTR: Variable-number tandem repeat analysis

HGDI: Hunter-Gaston discrimination index

SNPs: Single-nucleotide polymorphisms

WGS: Whole-genome Sequencing

MST: Minimum-spanning tree

\section{References}

1. Zhang, N.; Huang, D.; Wu, W.; Liu, J.; Liang, F.; Zhou, B.; Guan, P. Animal brucellosis control or eradication programs worldwide: A systematic review of experiences and lessons learned. Prev. Vet. Med. 2018, 160, 105-115, doi:10.1016/j.prevetmed.2018.10.002.

2. Uzureau, S.; Lemaire, J.; Delaive, E.; Dieu, M.; Gaigneaux, A.; Raes, M.; De Bolle, X.; Letesson, J.J. Global analysis of quorum sensing targets in the intracellular pathogen Brucella melitensis $16 \mathrm{M}$. J. Proteome Res. 2010, 9, 3200-3217, doi:10.1021/pr100068p.

3. Bagheri Nejad, R.; Krecek, R.C.; Khalaf, O.H.; Hailat, N.; Arenas-Gamboa, A.M. Brucellosis in the Middle East: Current situation and a pathway forward. PLoS Negl Trop Dis 2020, 14, e0008071, doi:10.1371/journal.pntd.0008071.

4. Rowe, M.T. BRUCELLA I Problems with Dairy Products. Encyclopedia of Food Microbiology 2014, 340343.

5. Ledwaba, M.B.; Gomo, C.; Lekota, K.E.; Le Flèche, P.; Hassim, A.; Vergnaud, G.; van Heerden, H. Molecular characterization of Brucella species from Zimbabwe. PLoS Negl Trop Dis 2019, 13, e0007311, doi:10.1371/journal.pntd.0007311.

6. Shang, D.; Xiao, D.; Yin, J. Epidemiology and control of brucellosis in China. Veterinary Microbiology 2002, 90, 165-182. 
7. Wang, H.; Xu, W.M.; Zhu, K.J.; Zhu, S.J.; Zhang, H.F.; Wang, J.; Yang, Y.; Shao, F.Y.; Jiang, N.M.; Tao, Z.Y., et al. Molecular investigation of infection sources and transmission chains of brucellosis in Zhejiang, China. Emerg Microbes Infect 2020, 10.1080/22221751.2020.1754137, 1-322, doi:10.1080/22221751.2020.1754137.

8. Sun, M.; Liu, M.; Zhang, X.; Zhang, G.; Zhu, L.; Ding, J.; Zhang, Z.; Sun, S.; Sun, S.; Shao, W., et al. First identification of a Brucella abortus biovar 4 strain from yak in Tibet, China. Vet. Microbiol. 2020, 247, 108751, doi:10.1016/j.vetmic.2020.108751.

9. Ran, X.; Cheng, J.; Wang, M.; Chen, X.; Wang, H.; Ge, Y.; Ni, H.; Zhang, X.X.; Wen, X. Brucellosis seroprevalence in dairy cattle in China during 2008-2018: A systematic review and meta-analysis. Acta Trop. 2019, 189, 117-123, doi:10.1016/j.actatropica.2018.10.002.

10. Zeng, J.Y.; Robertson, I.D.; Ji, Q.M.; Dawa, Y.L.; Bruce, M. Evaluation of the economic impact of brucellosis in domestic yaks of Tibet. Transbound Emerg Dis 2019, 66, 476-487, doi:10.1111/tbed.13049.

11. Al Dahouk, S.; Scholz, H.C.; Tomaso, H.; Bahn, P.; Göllner, C.; Karges, W.; Appel, B.; Hensel, A.; Neubauer, H.; Nöckler, K. Differential phenotyping of Brucella species using a newly developed semi-automated metabolic system. BMC Microbiol. 2010, 10, 269, doi:10.1186/1471-2180-10-269.

12. Liu, Z.G.; Wang, H.; Wang, M.; Li, Z.J. Investigation of the molecular epizootiological characteristics and tracking of the geographical origins of Brucella canis strains in China. Transbound Emerg Dis 2020, 67, 834843, doi:10.1111/tbed.13404.

13. Liu, Z.G.; Wang, L.J.; Piao, D.R.; Wang, M.; Liu, R.H.; Zhao, H.Y.; Cui, B.Y.; Jiang, H. Molecular Investigation of the Transmission Pattern of Brucella suis 3 From Inner Mongolia, China. Front Vet Sci 2018, 5, 271, doi:10.3389/fvets.2018.00271.

14. Ashford, R.T.; Muchowski, J.; Koylass, M.; Scholz, H.C.; Whatmore, A.M. Application of Whole Genome Sequencing and Pan-Family Multi-Locus Sequence Analysis to Characterize Relationships Within the Family Brucellaceae. Front Microbiol 2020, 11, 1329, doi:10.3389/fmicb.2020.01329.

15. Georgi, E.; Walter, M.C.; Pfalzgraf, M.T.; Northoff, B.H.; Holdt, L.M.; Scholz, H.C.; Zoeller, L.; Zange, S.; Antwerpen, M.H. Whole genome sequencing of Brucella melitensis isolated from 57 patients in Germany reveals high diversity in strains from Middle East. PLoS ONE 2017, 12, e0175425, doi:10.1371/journal.pone.0175425.

16. Ma, S.Y.; Liu, Z.G.; Zhu, X.; Zhao, Z.Z.; Guo, Z.W.; Wang, M.; Cui, B.Y.; Li, J.Y.; Li, Z.J. Molecular epidemiology of Brucella abortus strains from cattle in Inner Mongolia, China. Prev Vet Med 2020, 183, 105080, doi:10.1016/j.prevetmed.2020.105080.

17. Alton, G.G.; Jones, L.M.; Pietz, D.E. Laboratory techniques in brucellosis. Monogr Ser World Health Organ 1975, 1-163.

18. Al Dahouk, S.; Tomaso, H.; Nockler, K.; Neubauer, H.; Frangoulidis, D. Laboratory-based diagnosis of brucellosis--a review of the literature. Part I: Techniques for direct detection and identification of Brucella spp. Clin Lab 2003, 49, 487-505.

19. OIE. OIE Terrestrial manual; OIE: 2018; Vol. Part 3 OIE Listed Diseases and Other Diseases of Importance, pp. 44.

20. Bricker, B.J.; Halling, S.M. Differentiation of Brucella abortus bv. 1, 2, and 4, Brucella melitensis, Brucella ovis, and Brucella suis bv. 1 by PCR. J. Clin. Microbiol. 1994, 32, 2660-2666, doi:10.1128/jcm.32.11.26602666.1994.

21. Al Dahouk, S.; Flèche, P.L.; Nöckler, K.; Jacques, I.; Grayon, M.; Scholz, H.C.; Tomaso, H.; Vergnaud, G.; Neubauer, H. Evaluation of Brucella MLVA typing for human brucellosis. J. Microbiol. Methods 2007, 69, 137-145, doi:10.1016/j.mimet.2006.12.015.

22. Kattar, M.M.; Jaafar, R.F.; Araj, G.F.; Le Flèche, P.; Matar, G.M.; Abi Rached, R.; Khalife, S.; Vergnaud, G. Evaluation of a multilocus variable-number tandem-repeat analysis scheme for typing human Brucella isolates in a region of brucellosis endemicity. J. Clin. Microbiol. 2008, 46, 3935-3940, doi:10.1128/jcm.0046408.

23. Scholz, H.C.; Vergnaud, G. Molecular characterisation of Brucella species. Revue scientifique et technique 2013, 32, 149-162.

24. Hunter, P.R. Reproducibility and indices of discriminatory power of microbial typing methods. J Clin Microbiol 1990, 28, 1903-1905.

25. Liu, Z.G.; Wang, H.; Wang, M.; Li, Z.J. Investigation of the molecular epizootiological characteristics and tracking of the geographical origins of Brucella canis strains in China. Transbound Emerg Dis 2019, 10.1111/tbed.13404, doi:10.1111/tbed.13404. 
26. De Massis, F.; Ancora, M.; Atzeni, M.; Rolesu, S.; Bandino, E.; Danzetta, M.L.; Zilli, K.; Di Giannatale, E.; Scacchia, M. MLVA as an Epidemiological Tool To Trace Back Brucella melitensis Biovar 1 Re-Emergence in Italy. Transbound Emerg Dis 2015, 62, 463-469, doi:10.1111/tbed.12397.

27. Nascimento, M.; Sousa, A.; Ramirez, M.; Francisco, A.P.; Carrico, J.A.; Vaz, C. PHYLOViZ 2.0: providing scalable data integration and visualization for multiple phylogenetic inference methods. Bioinformatics 2016, 10.1093/bioinformatics/btw582, doi:10.1093/bioinformatics/btw582.

28. Vilella, A.J.; Severin, J.; Ureta-Vidal, A.; Heng, L.; Durbin, R.; Birney, E. EnsemblCompara GeneTrees: Complete, duplication-aware phylogenetic trees in vertebrates. Genome Res 2009, 19, 327-335, doi:10.1101/gr.073585.107.

29. Zhu, X.; Zhao, Z.; Ma, S.; Guo, Z.; Wang, M.; Li, Z.; Liu, Z. Brucella melitensis, a latent "travel bacterium," continual spread and expansion from Northern to Southern China and its relationship to worldwide lineages. 2020, 9, 1618-1627, doi:10.1080/22221751.2020.1788995.

30. Zhu, X.; Zhao, Z.; Ma, S.; Guo, Z.; Wang, M.; Li, Z.; Liu, Z. Brucella melitensis, a latent "travel bacterium," continual spread and expansion from Northern to Southern China and its relationship to worldwide lineages. Emerg Microbes Infect 2020, 9, 1618-1627, doi:10.1080/22221751.2020.1788995.

31. Yespembetov, B.A.; Syrym, N.S.; Zinina, N.N.; Sarmykova, M.K.; Konbayeva, G.M.; Basybekov, S.Z.; Mussayeva, A.K.; Kanatbayev, S.G.; Bazarbayev, M.; Siyabekov, S.T. Phenotypic and genotypic characteristics of Brucella isolates from the Republic of Kazakhstan. Trop Anim Health Prod 2019, 51, 23612370, doi:10.1007/s11250-019-01941-y.

32. Vergnaud, G.; Hauck, Y.; Christiany, D.; Daoud, B.; Pourcel, C.; Jacques, I.; Cloeckaert, A.; Zygmunt, M.S. Genotypic Expansion Within the Population Structure of Classical Brucella Species Revealed by MLVA16 Typing of 1404 Brucella Isolates From Different Animal and Geographic Origins, 1974-2006. Front Microbiol 2018, 9, 1545, doi:10.3389/fmicb.2018.01545.

33. De Massis, F.; Zilli, K.; Di Donato, G.; Nuvoloni, R.; Pelini, S.; Sacchini, L.; D'Alterio, N.; Di Giannatale, E. Distribution of Brucella field strains isolated from livestock, wildlife populations, and humans in Italy from 2007 to 2015. PLoS One 2019, 14, e0213689, doi:10.1371/journal.pone.0213689.

34. Sanogo, M.; Abatih, E.; Thys, E.; Fretin, D.; Berkvens, D.; Saegerman, C. Importance of identification and typing of Brucellae from West African cattle: a review. Vet Microbiol 2013, 164, 202-211, doi:10.1016/j.vetmic.2013.02.009.

35. Liu, Z.G.; Di, D.D.; Wang, M.; Liu, R.H.; Zhao, H.Y.; Piao, D.R.; Tian, G.Z.; Fan, W.X.; Jiang, H.; Cui, B.Y., et al. MLVA Genotyping Characteristics of Human Brucella melitensis Isolated from Ulanqab of Inner Mongolia, China. 2017, 8, 6, doi:10.3389/fmicb.2017.00006.

36. Garofolo, G.; Di Giannatale, E.; De Massis, F.; Zilli, K.; Ancora, M.; Cammà, C.; Calistri, P.; Foster, J.T. Investigating genetic diversity of Brucella abortus and Brucella melitensis in Italy with MLVA-16. Infect Genet Evol 2013, 19, 59-70, doi:10.1016/j.meegid.2013.06.021.

37. Sanogo, M.; Fretin, D.; Thys, E.; Saegerman, C. Exploring the Diversity of Field Strains of Brucella abortus Biovar 3 Isolated in West Africa. Front Microbiol 2017, 8, 1232, doi:10.3389/fmicb.2017.01232.

38. Bricker, B.J.; Ewalt, D.R. Evaluation of the HOOF-Print assay for typing Brucella abortus strains isolated from cattle in the United States: results with four performance criteria. BMC Microbiol 2005, 5, 37, doi:10.1186/1471-2180-5-37.

39. Kiliç, S.; Ivanov, I.N.; Durmaz, R.; Bayraktar, M.R.; Ayaslioglu, E.; Uyanik, M.H.; Aliskan, H.; Yasar, E.; Bayramoglu, G.; Arslantürk, A., et al. Multiple-locus variable-number tandem-repeat analysis genotyping of human Brucella isolates from Turkey. J Clin Microbiol 2011, 49, 3276-3283, doi:10.1128/jcm.02538-10.

40. Cosmo, N.D. Ancient Xinjiang Between Central Asia and China; The Nomadic Factor. Anthropology \& Archeology of Eurasia 1996, 34, 87-101, doi:10.2753/aae1061-1959340487.

41. Frachetti, M.D.; Smith, C.E.; Traub, C.M.; Williams, T. Nomadic ecology shaped the highland geography of Asia's Silk Roads. Nature 2017, 543, 193-198, doi:10.1038/nature21696.

42. Shevtsova, E.; Vergnaud, G.; Shevtsov, A.; Shustov, A.; Berdimuratova, K.; Mukanov, K.; Syzdykov, M.; Kuznetsov, A.; Lukhnova, L.; Izbanova, U., et al. Genetic Diversity of Brucella melitensis in Kazakhstan in Relation to World-Wide Diversity. Front Microbiol 2019, 10, 1897, doi:10.3389/fmicb.2019.01897.

43. Li, Z.; Wang, X.M.; Zhu, X.; Wang, M.; Cheng, H.; Li, D.; Liu, Z.G. Molecular Characteristics of Brucella Isolates Collected From Humans in Hainan Province, China. Front Microbiol 2020, 11, 452, doi:10.3389/fmicb.2020.00452. 
44. Garofolo, G.; Di Giannatale, E.; Platone, I.; Zilli, K.; Sacchini, L.; Abass, A.; Ancora, M.; Cammà, C.; Di Donato, G.; De Massis, F., et al. Origins and global context of Brucella abortus in Italy. BMC Microbiol. 2017, 17, 28, doi:10.1186/s12866-017-0939-0.

45. Tsend, S.; Baljinnyam, Z.; Suuri, B.; Dashbal, E.; Oidov, B.; Roth, F.; Zinstag, J.; Schelling, E.; Dambadarjaa, D. Seroprevalence survey of brucellosis among rural people in Mongolia. Western Pac Surveill Response J 2014, 5, 13-20, doi:10.5365/wpsar.2014.5.1.002.

46. Kang, S.I.; Her, M.; Erdenebaataar, J.; Vanaabaatar, B.; Cho, H.; Sung, S.R.; Lee, J.J.; Jung, S.C.; Park, Y.H.; Kim, J.Y. Molecular epidemiological investigation of Brucella melitensis circulating in Mongolia by MLVA16. Comp Immunol Microbiol Infect Dis 2017, 50, 16-22, doi:10.1016/j.cimid.2016.11.003.

47. Kulakov Iu, K.; Erdenebaator, J.; Tsirelson, L.E.; Tolmacheva, T.A.; Zheludkov, M.M.; Korenberg, E.I. [Molecular genetics typing of Brucella circulating in several provinces of Mongolia]. Zh Mikrobiol Epidemiol Immunobiol 2010, 17-22.

48. Sannikova, I.V.; Makhinya, O.V.; Maleev, V.V.; Deineka, D.A.; Golub, O.G.; Kovalchuk, I.V.; Lyamkin, G.I. Brucellosis in the Stavropol Territory: Results of 15-year follow-up of epidemiological and clinical features. Ter Arkh 2015, 87, 11-17, doi:10.17116/terarkh2015871111-17.

49. Alamian, S.; Dadar, M.; Wareth, G. Role of Brucella abortus Biovar 3 in the Outbreak of Abortion in a Dairy Cattle Herd Immunized with Brucella abortus Iriba Vaccine. Arch Razi Inst 2020, 75, 377-384, doi:10.22092/ari.2019.125468.1305. 